\title{
LIGHT-INDUCED TAUTOMERIZATION IN PORPHYRIN ISOMERS
}

\author{
J. WALUK \\ Institute of Physical Chemistry, Polish Academy of Sciences \\ Kasprzaka 44/52, 01-224 Warsaw, Poland
}

\begin{abstract}
Double proton tautomerization occurring in porphyrin and its structural isomers represents a special case of a chemical transformation in which the substrate and the product are formally the same. The methods used for the investigation of this kind of processes are based on polarized spectroscopy and high-resolution techniques, such as matrix isolation. Their combined use results in obtaining information pertinent to the mechanism of proton transfer, regarding e.g., the values of proton transfer rates, structure of the tautomeric forms or the shape of the potential energy surfaces. In addition, these procedures provide a way of obtaining spectral, photophysical and structural data that would be otherwise difficult to gain. The examples include determination of transition moment directions, assignment of electronic and vibrational states, elucidation of the character of the substitutional replacement of the rare gas matrix atoms by the chromophore, and the analysis of the nature of the symmetry lowering due to the matrix cage.

PACS numbers: 33.20.-t, 33.50.-j, 78.55.-m, 78.60.-b
\end{abstract}

\section{Introduction}

An apparently simple chemical reaction - transfer of two inner protons between the nitrogen atoms in porphyrin (1) - remains an object of intense studies, both by theoretical and experimental methods [1-43]. The reaction was found to proceed not only in the dark, but also after photoexcitation. The former process is practically stopped at around $100 \mathrm{~K}$, whereas the latter occurs even at liquid helium temperatures. Many basic questions regarding the reaction mechanism have not been answered, in particular those concerning the photoinduced process. Thus, the shape of the potential energy surface remains to be characterized, both qualitatively and quantitatively. There is no doubt that the dominant tautomeric species corresponds to the trans form, with protons residing on the opposite nitrogen atoms (1a in Fig. 1). However, the presence of metastable intermediates - the $c i s$ structures, where the protons occupy the positions on the adjacent nitrogen atoms (1 $\mathbf{b}$ in Fig. 1) was also postulated [21, 30]. Moreover, the experimental data suggest that the shape of the potential energy surface need not remain the same in different electronic states. Thus, a large difference is to 
be expected between the tautomerization rate constants in $S_{0}, S_{1}$, and $T_{1}$ levels. Only the ground state tautomerization rates have been so far carefully measured for $1[6,13,15,18,20-22,29-30]$. The reaction proceeds in $S_{0}$ as a thermally activated tunnelling process, involving two successive single-hydrogen transfers. The role of thermal activation vs. tunnelling in the photoinduced reaction remains to be characterized. Finally, it has yet to be determined whether the movement of the two protons in the excited state occurs in an asynchronous or a stepwise fashion.

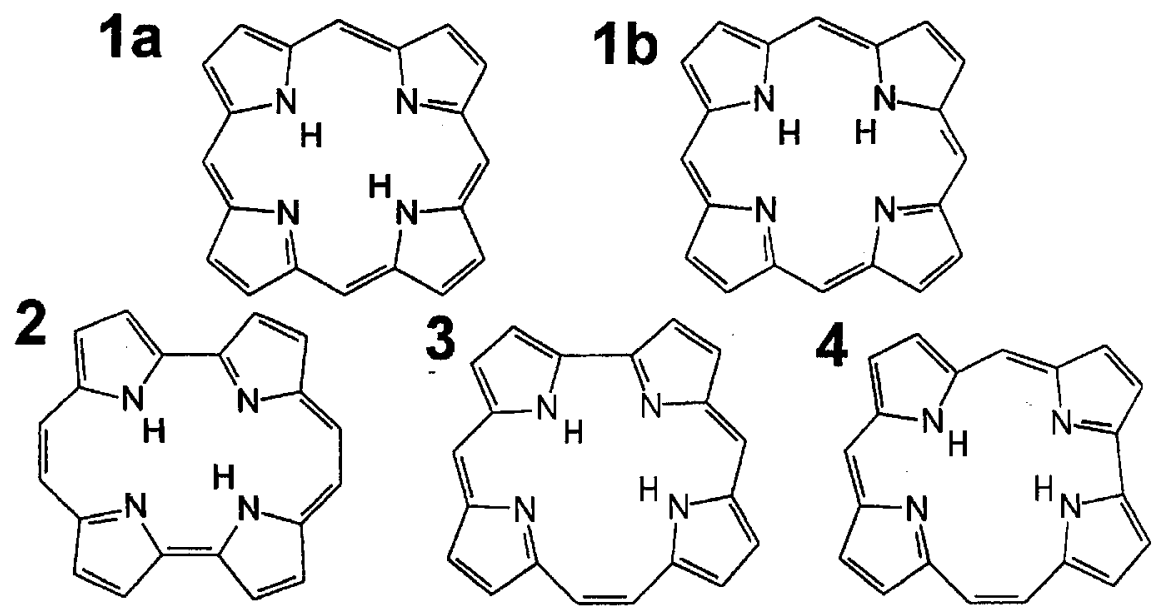

Fig. 1. Porphyrin in its trans (1a) and cis (1b) tautomeric forms, porphycene (2), corrphycene (3) and hemiporphycene (4). Thus far, 3 and 4 have only been obtained as octaalkyl derivatives.

Recent syntheses of structural isomers of porphyrin - porphycene (2) [44], corrphycene (3) [45] and hemiporphycene (4) [46] (Fig. 1) may turn out to be of great help in the study of tautomerization mechanisms. These new molecules, while differing from 1 with regard to symmetry and the NH-N separation, retain the electronic structure of porphyrin, and thus reveal its characteristic pattern of the electronic transitions - the presence of the analogues of the $Q$ and Soret bands. The molecules are planar, with well-defined values of the N-N distance. These properties make porphyrin isomers very attractive objects for studies of tautomerization.

A unique feature of the tautomerization process in 1-3 is that it formally transforms the molecule into itself (as long as it does not involve trans $\leftrightarrow$ cis interconversion). Therefore, special methods are required for the investigation of such a reaction. In this work, the application of two experimental techniques, polarized spectroscopy and matrix isolation, to the studies of tautomerization will be discussed, with a particular emphasis on the spectroscopic, photophysical, and structural information that can be extracted from such type of measurements in addition to the data regarding the proton transfer itself. 


\section{Polarized spectroscopy applied to the studies of proton transfer}

\subsection{Photoorientation}

The phenomenon of photoselection - obtaining an anisotropic ensemble of molecules after excitation - may be used for achieving permanent photoorientation - a long-lasting alignment of the sample. The idea is explained in Fig. 2. The sample, consisting of molecules that may undergo a photochemical transformation leading to a change in the position of the transition moment direction is irradiated, preferably with linearly polarized light, in a rigid matrix. The latter requirement ensures that the chromophore may not rotate, and the position of the molecular skeleton is fixed in space. Though the molecular skeleton does not move, the result of a photochemical process, such as a double proton transfer that may.occur in 1-3, is formally the same as if the molecule were rotated with respect to its environment. Therefore, the phenomenon is called pseudorotation. For instance, trans-trans tautomerization in 1 corresponds to a rotation by $90^{\circ}$ about an axis perpendicular to the molecular plane. In 2 and 3, because of lower symmetry, this angle may be smaller, depending on the absolute orientation of the transition moment directions in the molecular plane. This is an important result, which makes it possible to determine the transition moment directions in low-symmetry chromophores.

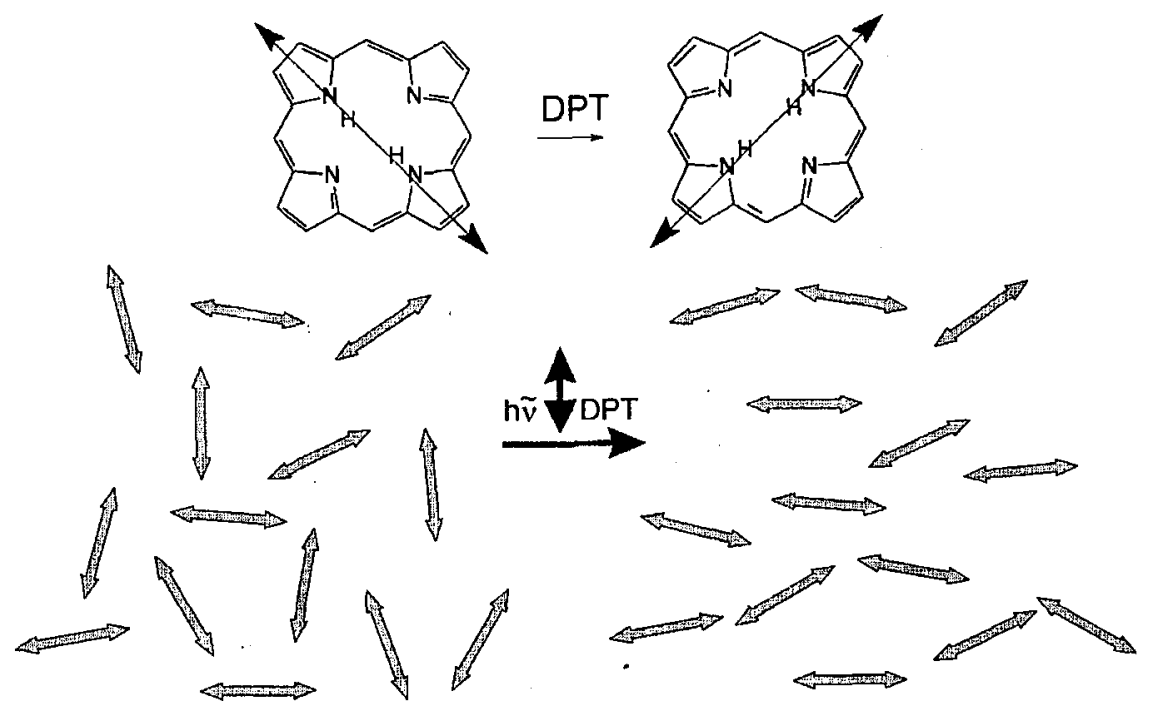

Fig. 2. Schematic principle of photoorientation: left, the isotropic sample in a rigid matrix; right, the partially aligned sample obtained after photoexcitation with linearly polarized light, followed by the double proton transfer (DPT) reactions. Double-headed arrows indicate the directions of the transition moment corresponding to the wavelength of excitation. For simplicity, only those molecules are represented whose planes lie in the plane of the drawing. 
Upon returning to the ground state, the product of the photoinduced process reveals a different orientation of its transition moment with respect to the electric vector of the photoexciting light. Since the probability of absorption varies as the cosine square of the angle between the transition moment direction and the electric vector of light, the molecules which have reacted will not absorb light to the same degree as before. In particular, for these porphyrin molecules, which had their transition moments parallel to the electric vector of light, the two vectors become orthogonal after tautomerization. Such molecules will no longer absorb at the wavelength used for phototransformation. In general, in the molecules that have reacted the transition moment corresponding to the $\pi \pi^{*}$ excitation, and all the transition moments parallel to it (not only those of electronic transitions, but also, e.g., those of the vibrational ones) will be tilted away from the electric vector of light.

As long as the molecules do not undergo a back reaction in the ground state, the photoorientation is permanent and may be studied, e.g., by measurements of linear dichroism (LD). The analysis of linear dichroism of photooriented samples has been worked out theoretically $[47,48]$ and applied in the experimental studies of porphyrin embedded in low-temperature rare gas matrices. An accurate determination of the transition moment directions was possible for both vibrational [31,34] and electronic [32] spectra. Thus, photoorientation is an efficient and elegant way of inducing alignment in a perfectly isotropic matrix. In this respect, it differs from standard methods of alignment, in which the orientation of the chromophore is due to the anisotropy of the matrices (stretched polymers, liquid crystals, flow cells, etc.)

\subsection{Luminescence depolarization and transient linear dichroism}

It should be realized that for achieving permanent photoorientation, large quantum yields of the phototransformation are not necessary. For irstance, the efficiency of phototransformation of $\mathbf{1}$ in Shpolskii matrices was estimated to be about $1 \%$ [26]. What is needed in such cases is the patience of the experimentalist, if a lamp is used as a light source. With a laser at hand, the effect can be observed in several minutes, or even less. Naturally, it is essential that the dark reaction does not occur. If it does, the tautomerization may be studied by using time-resolved polarization techniques, such as transient linear dichroism. The same method can be applied to the investigations of fast processes occurring in the excited states; for this case also stationary measurements of emission anisotropy may be successful. The principle is illustrated in Fig. 3. Again, a sample in a rigid environment is considered. Photoselection creates anisotropic populations both in the excited and in the ground state. If the reaction is faster than the lifetime of the transient species, the anisotropy will be changing with the rate characteristic of the process. Monitoring these changes by transient LD or time-resolved emission anisotropy can thus provide the values of the rate constants for the processes occurring both in the excited and in the ground state. For the latter, the time window available to the experiment is determined by the rate of ground state recovery. The upper limit for the time range of the ground state processes that can be studied in a 

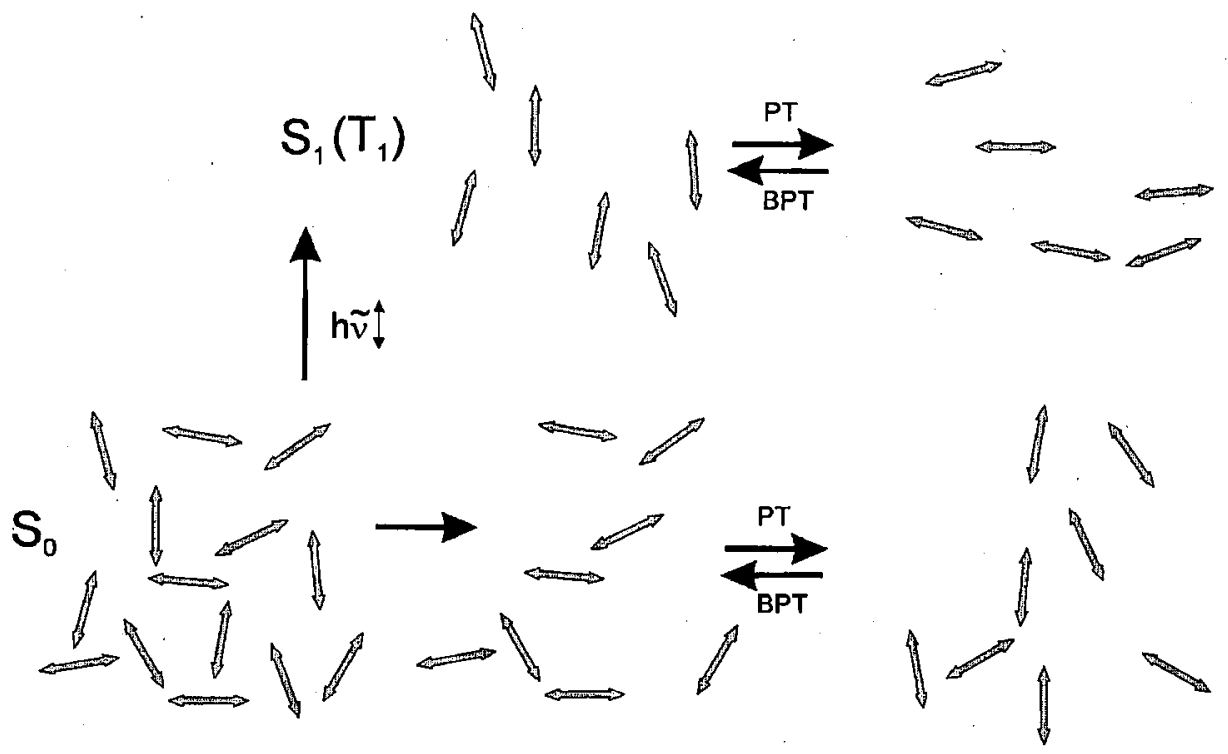

Fig. 3. Bottom left, the isotropic sample in a rigid matrix; middle, anisotropic populations in the excited and the ground state (top and bottom, respectively), obtained as a result of photoselection. Right, changes of molecular orientation due to the proton transfer reaction, resulting in the decrease in anisotropy. See caption to Fig. 2.

particular case depends on how significant is the fraction of molecules trapped in a long-lived triplet state, and on the lifetime of the latter.

Fluorescence of 1 does not reveal depolarization. The same is true for the octaethyl-substituted form of 4 [49]. On the contrary, the fluorescence of 2 was found to be depolarized in rigid glasses $[50,51]$. The same phenomenon was recently observed for the octaethyl-substituted form of 3 [52]. A proof was thus obtained for a rapid tautomerization occurring in the excited state of the latter two molecules. Since the fluorescence lifetimes are of the order of $10 \mathrm{~ns}$, the tautomerization rate must be faster than $10^{8} \mathrm{~s}^{-1}$. Recent results for porphycene in polymer matrices indicate that at temperatures below $20 \mathrm{~K}$ the rate of excited state tautomerization becomes slower than the $S_{1}$ lifetime [53]. A trans form must be involved in the process: if only the cis structures were present, even a rapid proton transfer between them could not lead to depolarization, because it would not change the transition moment directions. The depolarization cannot also occur in a hypothetical symmetrical structure with the inner protons located symmetrically in-between the nitrogen atoms. Thus, the possibility of such species can be rejected.

An interesting result obtained from the study of various alkyl derivatives of porphycene was that the rate constant for the proton transfer strongly decreases with the increase in the N-N distance [51]. Phototautomerization in $S_{1}$ was found to occur at temperatures as low as $90 \mathrm{~K}$ in all but one derivative $-2,3,6,7,12,13,16,17$-octaethylporphycene, which had the largest $\mathrm{N}-\mathrm{N}$ separation among the investigated derivatives. Recent studies of the latter molecule in poly- 
mer films show that even at $293 \mathrm{~K}$ the fluorescence anisotropy values are not altered by proton transfer. Thus, in octaethylporphycene the reaction rate is smaller than $10^{8} \mathrm{~cm}^{-1}$ even at room temperature, whereas in the other porphycenes it is much faster even at liquid nitrogen temperatures. This may explain also why no depolarization of fluorescence (and thus no proton transfer) occurs in the lowest excited singlet state of porphyrin, since the $\mathrm{N}-\mathrm{N}$ distance in this case is even larger. In turn, lack of fast excited state proton transfer in the octaethylderivative of $\mathbf{4}$ may be due to the fact that in this molecule the two trans tautomers are not chemically identical.

Just recently, we have found evidence of the ground state proton transfer in porphycene isolated in low-temperature supersonic jet [54]. The origin of splitting of spectral lines into doublets observed in the fluorescence excitation spectrum was attributed to tunnelling. Interestingly, these results suggest that the barrier for double proton transfer is larger in $S_{1}$ than in $S_{0}$.

Apart from yielding information concerning the proton transfer rates and the shape of the potential energy surfaces, measurements of emission anisotropy can lead to the determination of the electronic transition moment directions, even in the case of low symmetry molecules, such as $\mathbf{2}$ or $\mathbf{3}$. For a fast excited state trans-trans interconversion, assuming that the emission intensities and positions of both trans forms are the same leads to the following formulae for the emission anisotropy, $r[51,55]$ :

$$
\begin{aligned}
& r(\alpha, \beta)=\frac{1}{2} r(\beta-\alpha)+\frac{1}{2} r(\beta+\alpha) \\
&=\left\{3\left[\cos ^{2}(\beta-\alpha)+\cos ^{2}(\beta+\alpha)\right]-2\right\} / 10 \\
& r(\alpha, \alpha)=\frac{1}{2} r(0)+\frac{1}{2} r(2 \alpha)=\left[3 \cos ^{2}(2 \alpha)+1\right] / 10,
\end{aligned}
$$

where

$$
r(\phi)=\left[3 \cos ^{2}(\phi)-1\right] / 5 .
$$

The angles $\alpha$ and $\beta$ define the position of transition moments corresponding to the emission and absorption, respectively (Fig. 4). Equation (2) is a special case of (1), obtained for the parallel orientation of the transition moments of the absorbing and emitting states (e.g., for absorption into $S_{1}$ ).

More general expressions are obtained if one allows for the existence of both trans and cis tautomeric forms

$$
\begin{aligned}
& r(2 \alpha, \beta, \gamma, \delta)=(1-e) r(2 \alpha, \beta)+g r(\delta-\gamma)+(e-g) r(\beta-\gamma) \text { for } g<e, \\
& r(2 \alpha, \beta, \gamma, \delta)=(1-g) r(2 \alpha, \beta)+e r(\delta-\gamma)+(g-e) r(2 \alpha, \delta) \text { for } g>e,
\end{aligned}
$$

$\gamma$ and $\delta$ are the analogues of $\alpha$ and $\beta$ in the cis forms (cf. Fig. 4). Due to symmetry, their values may only be $0^{\circ}$ or $90^{\circ} ; g$ and $e$ indicate the fractions of $c i s$ tautomers in the ground and excited state, respectively. In Eqs. (4) and (5) it is assumed that the interconversion between the trans tautomers is rapid in $S_{1}$ with respect to the excited state lifetime $\left(k_{\mathrm{tt}}>1 / \tau\left(S_{1}^{\mathrm{tr}}\right)\right.$, cf. Fig. 4$)$ and that all species emit with the same quantum yield. A cis-trans excited state tautomerization process is allowed to occur in one direction only, i.e. $k_{\mathrm{tc}} \gg k_{\mathrm{ct}}$ or $k_{\mathrm{ct}} \gg k_{\mathrm{tc}}$. 


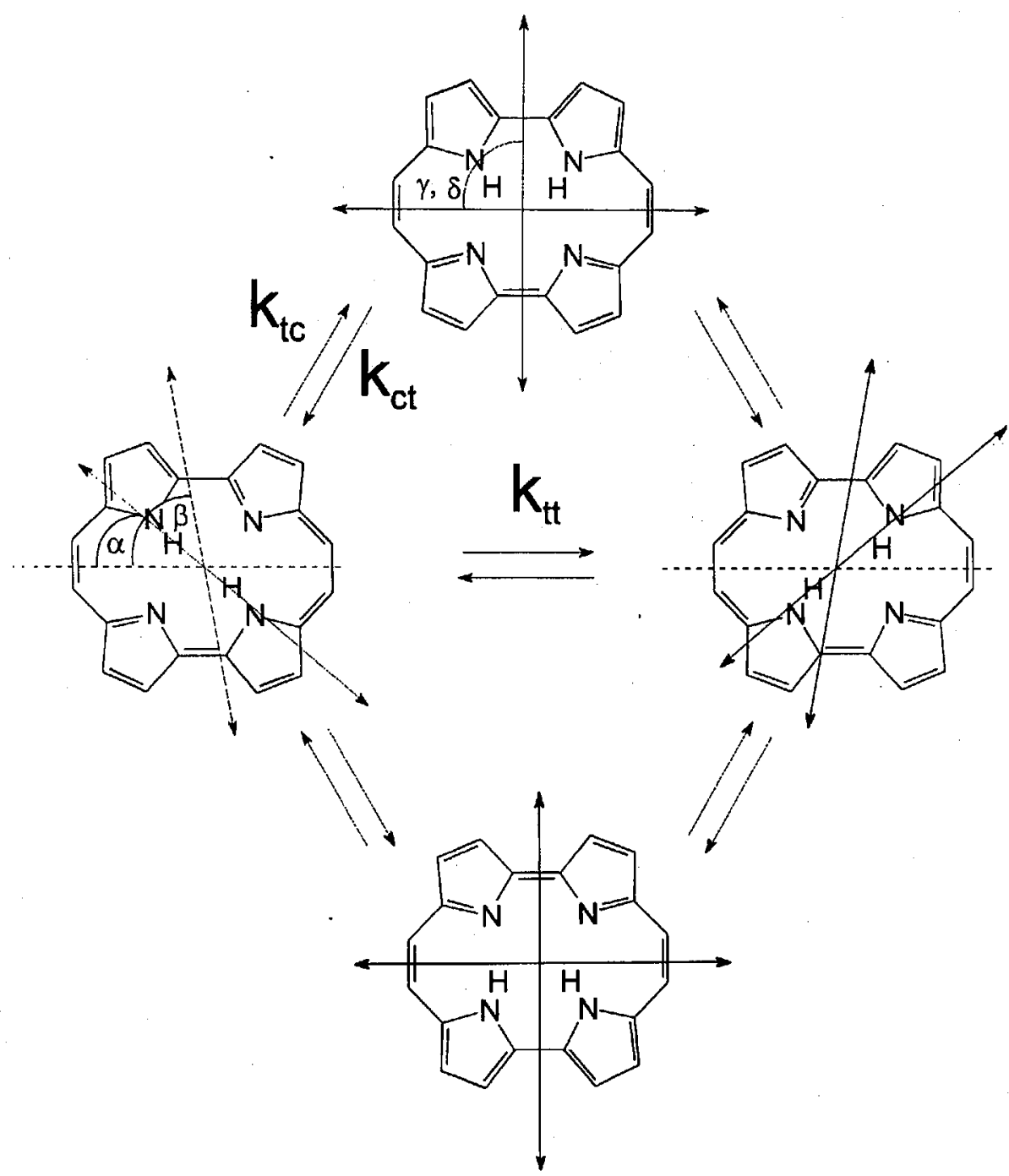

Fig. 4. Possible proton transfer equilibria in porphycene and the directions of transition moments in various tautomeric forms. $\alpha$ and $\gamma$ are the angles formed by the transition moment of the emitting state with the horizontal axis in the trans and cis tautomers, respectively; $\beta$ and $\delta$ are the corresponding angles for the absorbing states.

For the case when the interconversion between the cis and trans forms is very rapid as compared to the $S_{1}$ decay time, i.e. $k_{\mathrm{tt}}, k_{\mathrm{ct}}, k_{\mathrm{tc}}>1 / \tau\left(S_{1}\right)$, the fluorescence anisotropy is given by

$$
\begin{gathered}
r(2 \alpha, \beta, \gamma, \delta)=(1-g)(1-e) r(2 \alpha, \beta)+(1-g) \operatorname{er}(\gamma-\beta) \\
+g(1-e) r(2 \alpha, \delta)+g e r(\gamma-\delta) .
\end{gathered}
$$


Using Eqs. (1)-(5), transition moment directions in porphycene and several of its alkylated derivatives were determined from the measured fluorescence anisotropy values [51]. The procedure was cross-checked by comparing the results obtained from fluorescence anisotropy measurements with those provided by standard LD measurements performed in stretched polyethylene sheets. This was done for 9,10,19,20-tetra- $n$-propylporphycene, a molecule that, due to its elongated shape, aligns very well in stretched sheets [55]. Both methods gave very similar results, which also nicely agreed with the calculated transition moment directions. It is interesting to mention a certain correspondence between porphyrin, porphycene and corrphycene with regard to the transition moment directions. In $\mathbf{1}$, the transition moments corresponding to $S_{0}-S_{1}$ and $S_{0}-S_{2}$ transitions are strictly orthogonal: the former is parallel to the $\mathrm{NH}-\mathrm{HN}$ axis, the latter is perpendicular to it. In 2 , this pattern is also approximately obeyed, even though the molecule has a lower symmetry and all directions in the molecular plane are allowed. The initial results for octaethylcorrphycene [52] indicate that also this molecule reveals a similar pattern of the two lowest transition moment directions.

\section{Matrix isolation studies: the informational content of the multiplet structure (or of the lack of it)}

The formal identity of the two trans (or cis) tautomers in 1-3 is only valid for the isolated chromophores. Placing a molecule into a condensed phase may lead to a different interaction of each form with the matrix and thus, to spectral differences. This is indeed observed for rigid solvents which allow to observe well-resolved spectra: rare gases, Shpolski matrices, or polymer films. A very important feature revealed by 1 is that at low temperatures the tautomers may be reversibly phototransformed into each other. If the polarized light is used for the phototransformation, different characteristics of linear dichroism may be produced, depending on whether a broad or a narrow band irradiation source is used [31]. Comparison of the LD results obtained on samples photooriented using both techniques for achieving photoorientation provides yet another proof of the correctness of the model and leads to more accurate determination of transition moment directions.

The site splitting due to proton transfer has a different origin than the splitting caused by different substitutional replacements. The two interconverting tautomers occupy the same location in a matrix, but can be "pseudorotated" back and forth by photoexcitation. In other words, the ensemble of the same molecules may appear at different spectral locations, contrary to the standard situation, where different spectral lines are produced by different subsets of molecules.

The analysis of the patterns observed in high-resolution spectra is very useful for the state assignment. Porphyrin reveals a very characteristic multiplet structure in the electronic spectra, different for each electronic transition and each matrix. A large splitting of the lines belonging to two interconverting tautomers was obtained for the $S_{0}-S_{1}$ transition in xenon. The same pattern was detected in phosphorescence, providing a strong argument for the same orbital origin of the $S_{1}$ and $T_{1}$ states [33]. In order to reconcile this finding with the postulate that in frozen alkane matrices the $T_{1}$ state corresponds to $S_{2}$, not to $S_{1}$ [56], it has to be assumed that the two lowest triplet states of 1 lie close to each other, and that 
their ordering may be reversed by changing the environment. Given the important role of the porphyrin triplet state in the biological processes, further studies seem worthwhile.

A strong correlation was obtained between spectral lines observed in the electronic and infrared spectra $[31,57]$. Phototransformation between the tautomers leads to a decrease in the intensity of lines corresponding to the absorption of the substrate and to the increase in the intensity of lines corresponding to the absorption of the product. The same pattern is observed in the infrared spectrum. This makes possible to obtain a single site/single tautomer spectra both in the electronic and vibrational regions. Moreover, the magnitude and the sign of the site splitting due to proton transfer in the infrared region are strongly dependent on the form of the vibration and thus provide an additional tool for the assignment of vibrational states.

Another application of the analysis of site splitting is provided by the comparison of splittings observed in porphyrin with those measured for metalloporphyrins (Fig. 5). A close similarity is obtained in the absorption spectral pattern, but not in emission [58]. For the latter, the doublet splitting is observed in 1, but not in its metal derivatives. The reason is that in free base porphyrin, the doublet in absorption and emission arises because two tautomers interact differently with the matrix, and thus have different $S_{0}-S_{1}$ and $S_{0}-T_{1}$ transition energies. On the contrary, in metalloporphyrins, the components of the doublet observed in the absorption correspond to $S_{0}-S_{1}$ and $S_{0}-S_{2}$ transitions, respectively. Only the former is revealed in the emission and therefore, only one line is observed in fluorescence. The removal of the degeneracy of the $S_{0}-S_{1}$ and $S_{0}-S_{2}$ transitions is due to the matrix effect, apparently very similar for free base and metalloporphyrins. The implication is that the symmetry distortion in metalloporphyrins occurs along the $\mathrm{N}-\mathrm{N}$ axis, not along the line connecting the opposite meso carbon atoms.

Comparison of the site splitting patterns observed for porphyrin and porphycene (Fig. 5) reveals a much less rich structure in the latter. This may be due to different symmetries of the two compounds, and the resulting different directions of the transition moment vectors. For xenon and krypton matrices, the cavity required for the accommodation of 1 or 2 has an approximately hexagonal shape. The two trans tautomers of 1 may interact to a different degree with the matrix, whereas the two analogous forms of $\mathbf{2}$ should reveal similar interaction energies (Fig. 6). Thus, much smaller site splitting is expected for the latter case. This is indeed observed [59]. For the interconversion between two trans porphycene tautomers the transition moment of the $S_{0}-S_{1}$ in the substrate approximately coincides with the direction of the $S_{0}-S_{2}$ transition moment in the product, and vice versa. In consequence, the two transitions should reveal a similar site splitting pattern. This was observed, especially in argon, where the multiplet structure is quite rich. Moreover, the two transitions should be strongly correlated in the line narrowing experiments. In fact, our recent studies of 2 in a poly(vinyl butyral) sheets showed that the line narrowing effect is simultaneously observed for the region of $S_{0}-S_{1}$ and $S_{0}-S_{2}$ transitions, but not for higher excited states [53]. 


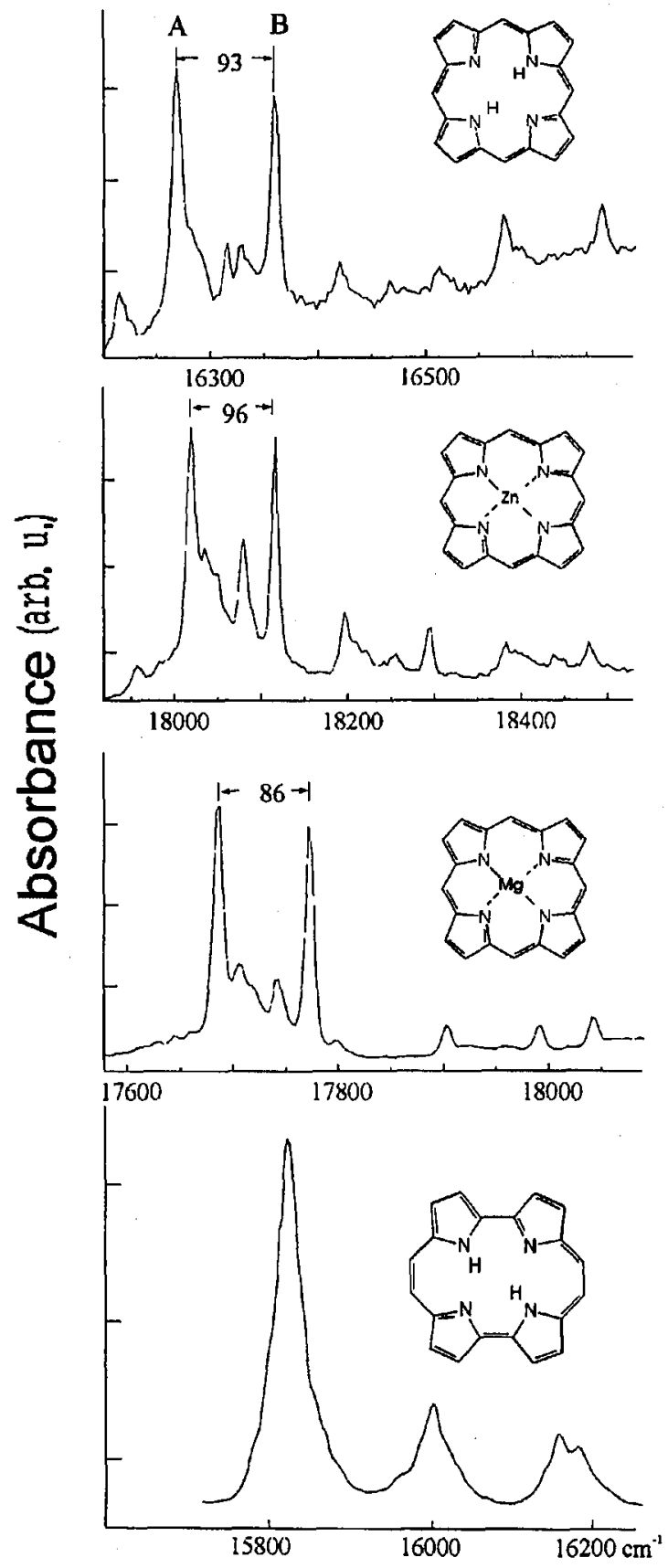

Fig. 5. Absorption spectra of free base, zinc and magnesium porphyrin, and of porphycene in the (0-0) region of the $S_{0}-S_{1}$ transition in Xe matrix at $15 \mathrm{~K}$. 


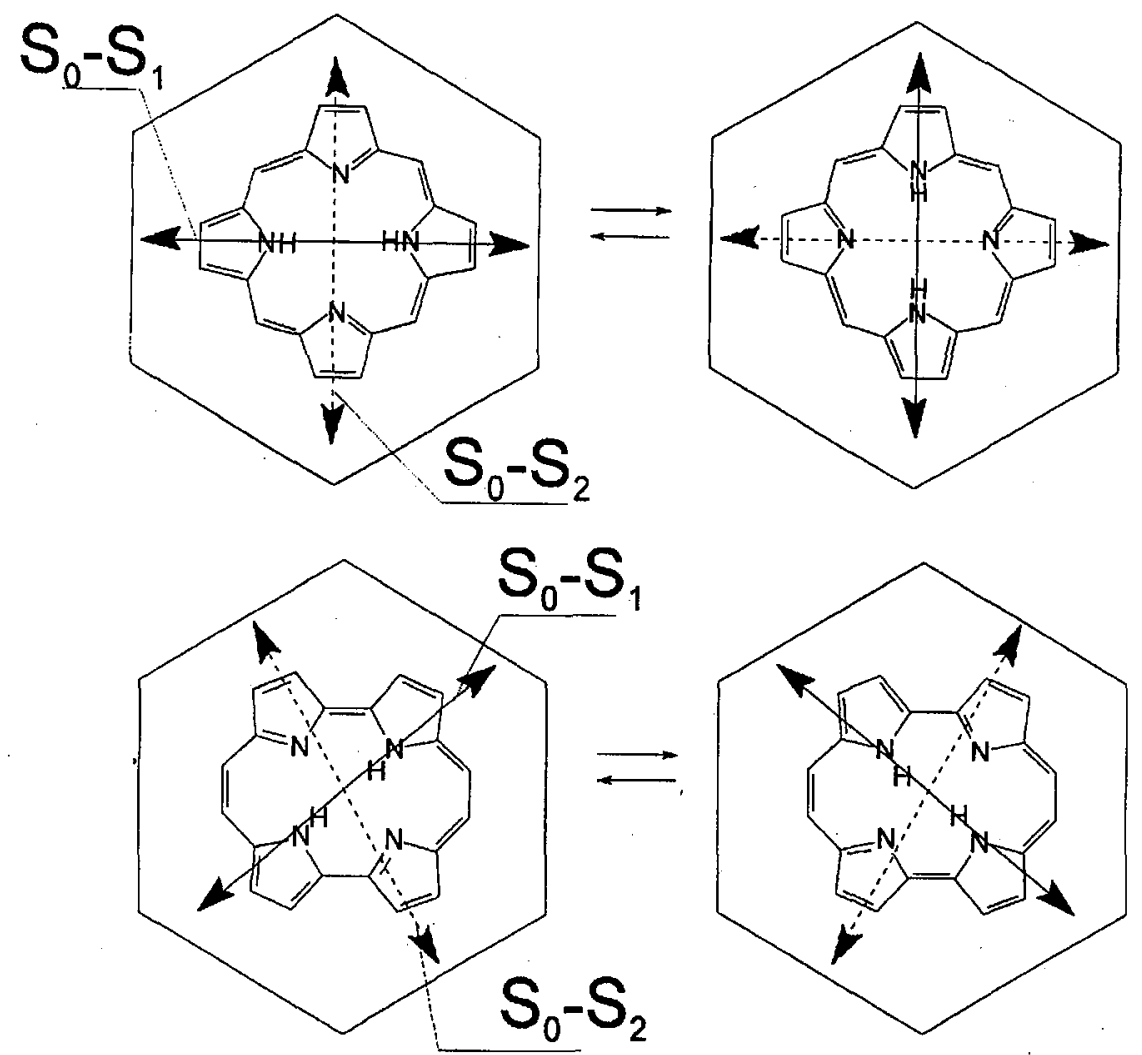

Fig. 6. Directions of $S_{0}-S_{1}$ and $S_{0}-S_{2}$ transition moments with respect to the matrix cage of hexagonal symmetry. Top - porphyrin, bottom - porphycene.

\section{Conclusions}

The methodology used for studying proton transfer processes provides not onfy the information concerning tautomerization itself, but also the access to spectral and structural data difficult to obtain by other techniques. The actual proton transfer can be used in this context as a very expedient tool that makes it possible to "pseudorotate" a molecule back and forth in a rigid environment by a well-defined angle.

The methods presented in this work can naturally be extended to the studies of other processes. Actually, it is not an exception but rather a rule that the transition moment direction of a reaction product is located differently from the transition moment of the substrate. Rigid phase photochemistry, coupled with the use of polarized light, may thus become a very useful general technique.

\section{Acknowledgments}

The work has been supported by the Committee for Scientific Research (grant 3T09A 063 14). 


\section{References}

[1] C.B. Storm, Y. Teklu, J. Am. Chem. Soc. 94, 1745 (1972); C.B. Storm, Y. Teklu, E.A. Sokoloski, Ann. N.Y. Acad. Sci. 206, 631 (1973).

[2] R.J. Abraham, G.E. Hawkes, K.M. Smith, Tetrahedron Lett. 16, 1483 (1974); J. Chem. Soc. Perkin Trans. 2, 1483 (1974).

[3] C.S. Irving, A. Lapidot, J. Chem. Soc. Chem. Commun., 184 (1977).

[4] H.J.C. Yeh, M. Sato, I.J. Morishima, Magn. Res. 26, 365 (1977).

[5] D. Gust, J.D. Roberts, J. Am. Chem. Soc. 99, 3637 (1977).

[6] S.S. Eaton, G.R. Eaton, J. Am. Chem. Soc. 113, 1601 (1977).

[7] P. Stilbs, M.E. Moseley, J. Chem. Soc. Faraday Trans. 276, 729 (1980); P. Stilbs, J. Magn. Res. 58, 152 (1984).

[8] J. Hennig, H.-H. Limbach, J. Chem. Soc. Faraday Trans. 2 75, 752 (1979); J. Magn. Res. 49, 322 (1982); J. Am. Chem. Soc. 106, 292 (1984).

[9] J. Braun, C. Hasenfranz, R. Schwesinger, H.-H. Limbach, Angew. Chem. Int. Ed. Engl. 33, 2215 (1994).

[10] H.-H. Limbach, J. Hennig, D. Gerritzen, H. Rumpel, Faraday Discuss. Chem. Soc. 74, 822 (1982).

[11] H.-H. Limbach, in: Dynamic NMR Spectrocopy in the Presence of Kinetic Isotope Effects, Vol. 23, NMR Basic Princ. Prog., Springer, Heidelberg 1990, Ch. 2.

[12] M. Schlabach; B. Wehrle, H.-H. Limbach, E. Bunnenberg, A. Knierziger, A.Y.L. Shu, B.R. Tolf, C. Djerassi, J. Am. Chem. Soc. 108, 3856 (1986).

[13] M. Schlabach, H.-H. Limbach, E. Bunnenberg, A.Y.L. Shu, B.R. Tolf, C. Djerassi, J. Am. Chem. Soc. 115, 4554 (1993).

[14] M. Schlabach, H. Rumpel, H.-H. Limbach, Angew. Chem. Int. Ed. Engl. 28, 76 (1989).

[15] M.L. Crossley, L.D. Field, M.M. Harding, S. Sternhell, J. Am. Chem. Soc. 109, 2335 (1987); M.L. Crossley, M.M. Harding, S. Sternhell, J. Am. Chem. Soc. 108, 3608 (1986).

[16] L. Frydman, A.C. Olivieri, L.E. Diaz, B. Frydman, F.G. Morin, C.L. Mayne, D.M. Grant, A.D. Adler, J. Am. Chem. Soc. 110, 336 (1988); L. Frydman, A.C. Olivieri, L.E. Diaz, B.A. Valasinas, B. Frydman, ibid. 110, 5661 (1988).

[17] H.-H. Limbach, J. Hennig, R. Kendrick, C.S. Yannoni, J. Am. Chem. Soc. 106, 4059 (1984); H.-H. Limbach, B. Wehrle, H. Zimmermann, R.D. Kendrick, C.S. Yannoni, ibid. 109, 929 (1987); Angew: Chem. Int. Ed. Engl. 26, 247 (1987); H.-H. Limbach, B. Wehrle, M. Schlabach, R.D. Kendrick, C.S. Yannoni, J. Magn. Res. 77, 84 (1988).

[18] B. Wehrle, H.-H. Limbach, M. Köcher, O. Ermer, E. Vogel, Angew. Chem. Int. Ed. Engl. 26, 934 (1987).

[19] B. Wehrle, H.-H. Limbach, H. Zimmermann, Ber. Bunsenges. Phys. Chem. 91, 941 (1987).

[20] M. Schlabach, B. Wehrle, H. Rumpel, J. Braun, G. Scherer, H.-H. Limbach, Ber. Bunsenges. Phys. Chem. 96, 821 (1992).

[21] J. Braun, M. Schlabach, B. Wehrle, M. Köcher, E. Vogel, H.-H. Limbach, J. Am. Chem. Soc. 116, 6593 (1996).

[22] J. Braun, H.-H. Limbach, P.G. Williams, H. Morimoto, D.E. Wemmer, J. Am. Chem. Soc. 118, 7231 (1996). 
[23] K.N. Solov'ev, I.E. Zalesski, V.N. Kotlo, S.F. Shkirman, Phys. Lett. 17, 332 (1973).

[24] I.E. Zalesski, V.N. Kotlo, A.N. Sevchenko, K.N. Solov'ev, S.F. Shkirman, Sov. Phys.-Dokl. (Eng. Transl.) 17, 1183 (1973).

[25] O.N. Korotaev, R.I. Personov, Opt. Spectrosc. (Engl. Transl.) 32, 479 (1972).

[26] S. Völker, J.H. van der Waals, Mol. Phys. 32, 1703 (1976).

[27] S. Völker, R.M. McFarlane, A.Z. Genack, H.P. Trommsdorff, J.H. van der Waals, J. Chem. Phys. 67, 1759 (1977).

[28] S. Völker, R.M. McFarlane, IBM J. Res. Dev. 23, 547 (1979).

[29] T.J. Butenhoff, R.S. Chuck, H.-H. Limbach, C.B. Moore, J. Phys. Chem. 94, 7847 (1990).

[30] T.J. Butenhoff, C.B. Moore, J. Am. Chem. Soc. 110, 8336 (1988).

[31] J. Radziszewski, J. Waluk, J. Michl, Chem. Phys. 136, 165 (1989).

[32] J. Radziszewski, J. Waluk, J. Michl, J. Mol. Spectrosc. 140, 373 (1990).

[33] J. Radziszewski, J. Waluk, M. Nepraš, J. Michl, J. Phys. Chem. 95, 1963 (1991).

[34] J. Radziszewski, M. Nepraš, V. Balaji, J. Waluk, E. Vogel, J. Michl, J. Phys. Chem. 99, 14254 (1995).

[35] J. Almlöf, Int. J. Quant. Chem. 8, 915 (1974); A. Ghosh, J. Almlöf, J. Phys. Chem. 99, 1073 (1995).

[36] H.-H. Limbach, J. Hennig, J. Chem. Phys. 71, 3120 (1979); H.-H. Limbach, ibid. 80, 5343 (1984); H.-H. Limbach, J. Hennig, J. Stulz, ibid. 78, 5432 (1983).

[37] G.I. Bersuker, V.Z. Polinger, Chem. Phys. 86, 57 (1984).

[38] A. Sarai, Chem. Phys. Lett. 83, 50 (1981); J. Chem. Phys. 76, 5554 (1982); ibid. 80, 5341 (1984).

[39] W:A. Kuz'mickij, A.N. Sevchenko, K.N. Solov'ev, Dokl. AN USSR 239, 308 (1978); V.A. Kusmitsky, K.N. Solovyov, J. Mol. Struct. 65, 219 (1980).

[40] Z. Smerdachina, W. Siebrand, T.A. Wildman, Chem. Phys. Lett. 143, 395 (1988);

Z. Smerdachina, W. Siebrand, F. Zerbetto, Chem. Phys. 136, 285 (1989).

[41] D.C. Rawlings, R.E. Davidson, M. Gouterman, Theor. Chim. Acta 61, 227 (1982).

[42] J.R. Reimers, T.X. Lü, M.J. Crossley, N.S. Hush, J. Am. Chem. Soc. 117, 2855 (1995).

[43] J. Baker, P.M. Kozlowski, A.A. Jarzecki, P. Pulay, Theor. Chem. Acc. 97, 59 (1997).

[44] E. Vogel, M. Köcher, H. Schmickler, J. Lex, Angew. Chem. Int. Ed. Engl. 25, 257 (1987).

[45] J.L. Sessler, E.A. Brucker, S.J. Weghorn, M. Kisters, M. Schäfer, J. Lex, E. Vogel, Angew. Chem. Int. Ed. Engl. 33, 2308 (1994); M.A. Aukauloo, R. Guilard, New. J. Chem. 18, 1205 (1994); H. Falk, Q.-Q. Chen, Monatsh. Chem. 69, 127 (1996).

[46] H.J. Callot, A. Rohrer, T. Tschamber, B. Metz, New. J. Chem. 19, 155 (1995); E. Vogel, M. Bröring, S.J. Weghorn, P. Scholz, R. Deponte, J. Lex, H. Schickler, K. Schaffner, S.E. Braslavsky, M. Müller, S. Pörting, C.J. Fowler, J.L. Sessler, Angew. Chem. Int. Ed. Engl. 36, 1651 (1997).

[47] J. Michl, E.W. Thulstrup, Spectroscopy with Polarized Light (paperback edition), VCH Publishers, New York 1995. 
[48] J.G. Radziszewski, F.A. Burkhalter, J. Michl, J. Am. Chem. Soc. 109, 67 (1981).

[49] P. Borowicz, J. Dobkowski, E. Vogel, J. Waluk, in preparation.

[50] J. Waluk, M. Müller, P. Swiderek, M. Köcher, E. Vogel, G. Hohlneicher, J. Michl, J. Am. Chem. Soc. 113, 5511 (1991).

[51] J. Waluk, E. Vogel, J. Phys. Chem. 48, 4530 (1994).

[52] P. Borowicz, E. Vogel, J. Waluk, in preparation.

[53] M. Gil, J. Waluk, unpublished results.

[54] J. Sepioł, Y. Stepanenko, A. Vdovin, J. Jasny, A. Mordziński, E. Vogel, J. Waluk, Chem. Phys. Lett. 296, 549 (1998).

[55] K.B. Andersen, E. Vogel, J. Waluk, Chem. Phys. Lett. 271, 341 (1997).

[56] W.G. Van Dorp, M. Soma, J.A. Kooter, J.H. van der Waals, Mol. Phys. 28, 1551 (1974):

[57] J. Radziszewski, J. Michl, J. Waluk, Proc. SPIE 1575, 606 (1991).

[58] A. Starukhin, A. Shulga, J. Waluk, Chem. Phys. Lett. 272, 405 (1997).

[59] A. Starukhin, E. Vogel, J. Waluk, J. Phys. Chem., in press. 